Revue internationale d'éducation de Sèvres

\title{
Repenser et refonder l'école au Maroc : la Vision stratégique 2015-2030
}

Rahma Bourqia

\section{OpenEdition}

12 Journals

Édition électronique

URL : https://journals.openedition.org/ries/4551

DOI : 10.4000/ries.4551

ISSN : 2261-4265

Éditeur

France Education international

\section{Édition imprimée}

Date de publication : 1 avril 2016

Pagination : 18-24

ISBN : 978-2-85420-610-4

ISSN : $1254-4590$

Référence électronique

Rahma Bourqia, «Repenser et refonder l'école au Maroc : la Vision stratégique 2015-2030 », Revue internationale d'éducation de Sèvres [En ligne], 71 | avril 2016, mis en ligne le 01 avril 2018, consulté le 01 juillet 2021. URL : http://journals.openedition.org/ries/4551 ; DOI : https://doi.org/10.4000/ries. 4551

Ce document a été généré automatiquement le 1 juillet 2021.

(c) Tous droits réservés 


\title{
Repenser et refonder l'école au Maroc : la Vision stratégique 2015-2030
}

\author{
Rahma Bourqia
}

1 Si de nombreux pays, à un moment donné de leur histoire, interrogent leur école et ses missions, le Maroc n'échappe pas à ces interrogations. L'évolution et le développement du système d'éducation et de formation marocain, organisé en niveaux primaire secondaire, professionnel et supérieur ont certainement contribué, depuis l'indépendance du Maroc en 1956, à produire des générations de scolarisés et de diplômés. Mais aujourd'hui, l'école fait objet d'interrogations sur sa capacité à se mettre au niveau des défis d'une éducation et formation du XXI ${ }^{e}$ siècle. En effet, l'aptitude de l'école à générer une éducation de qualité préparant les individus à affronter leur avenir est mise à l'épreuve par une demande sociale pressante. Ce qui a entraîné une réflexion profonde pour repenser l'école marocaine et la refonder dans le cadre de l'élaboration d'une nouvelle vision qui trace une nouvelle voie pour l'éducation.

\section{Les éléments du contexte d'une réforme}

2 La réforme de l'éducation lancée au Maroc survient dans un contexte où interagissent trois éléments : la pression sociale sur l'école, une nouvelle Constitution en 2011 et une évaluation de la mise en œuvre de la Charte nationale de l'éducation et de la formation mise en œuvre de 2000 à 2013.

\section{La pression sociale sur l'école}

3 Sans arriver à satisfaire les différentes attentes, l'école publique marocaine a évolué vers une crise pour devenir la cible de critiques provenant de différentes sources. La société interpelle l'école pour qu'elle soit le moteur et le miroir de son développement 
humain. L'État tend à voir dans l'éducation une réussite de sa politique. Les familles transposent sur l'école leurs aspirations pour qu'elle soit le vecteur qui réalise la mobilité sociale de leurs enfants, l'entreprise voudrait que l'école forme une main d'œuvre qualifiée et opérationnelle sur le marché de l'emploi, et les jeunes élèves et étudiants aspirent à ce que l'école leur offre le sésame du passage à l'âge adulte. C'est ainsi que l'école est sous la pression d'une multitude d'attentes variées et différenciées, dont les logiques ne convergent pas toujours.

\section{Une nouvelle Constitution en 2011}

4 Si le Maroc a connu depuis les années quatre-vingt-dix des réformes constitutionnelles et politiques, les mouvements, communément appelés "printemps arabe ", qui ont émergé avec des manifestations d'une jeunesse revendiquant plus de justice, de démocratie et de développement, ont accéléré le processus des changements en 2011 avec l'élaboration d'une nouvelle Constitution qui a apporté des éléments nouveaux et fondamentaux pour l'éducation. Parmi ces éléments, on trouve l'accès et le droit de chaque citoyen(ne) «à une éducation moderne, accessible et de qualité » (article 31) et le fait que «l'enseignement fondamental est un droit de l'enfant et une obligation de la famille et de l'État » (article 32). Le Conseil supérieur de l'éducation, de la formation et de la recherche scientifique. (CSEFRS) est instauré comme «instance de bonne gouvernance et de régulation", à laquelle est confiée une mission "consultative chargée d'émettre son avis sur toutes les politiques publiques et sur les questions d'intérêt national concernant l'éducation, la formation et la recherche scientifique, ainsi que sur les objectifs et le fonctionnement des services publics chargés de ces domaines. Ce Conseil contribue également à l'évaluation des politiques et programmes publics menés dans ces domaines" (article 168). C'est ainsi que le constituant lui assigne la charge de l'élaboration des orientations stratégiques, des avis et des évaluations pour impulser les réformes du système d'éducation, de formation et de recherche scientifique.

5 Le nouveau contexte créé par cette nouvelle constitution exige que le lien organique entre la Constitution, porteuse d'un nouveau projet de société, et le projet éducatif soit affirmé et annoncé à travers une réforme profonde de l'éducation et de la formation. L'idée directrice qui anime la nouvelle Vision est de concevoir des réformes qui reflètent les principes et les valeurs annoncés par le contrat social que représente la Constitution de 2011, au niveau de l'organisation et du fonctionnement du système d'éducation et de formation, des programmes pédagogiques ainsi que des méthodes d'apprentissage et de formation.

\section{Une évaluation de la mise en œuvre de la Charte 2000-2013}

6 C'est dans le cadre de la mise en place du CSEFRS, selon les nouvelles missions que lui attribuent la Constitution et la loi, qu'a émergé le projet de repenser l'école marocaine en réalisant une évaluation de la mise en œuvre de la Charte nationale d'éducation et de formation de 2000 à $2013^{1}$. Cette évaluation montre que malgré les progrès réalisés, qui reflètent la volonté d'appliquer les recommandations de la Charte, des défaillances persistent dans le processus de mise en œuvre. Elle a permis d'identifier les nœuds sur 
lesquels il faudrait agir pour rattraper le retard accumulé pour mettre à niveau le système éducatif.

7 Suite à cette évaluation, la Vision stratégique 2015-2030 a été élaborée. Ses recommandations comportent quatre axes majeurs pour refonder l'éducation : équité ; qualité ; émancipation de l'individu et développement de la société ; conduite du changement avec un leadership efficace. Ces axes ou «fondements » sont déclinés en 23 leviers et 134 dispositions pour orienter la mise en œuvre des réformes à entreprendre.

\section{Une nouvelle vision stratégique $2015-2030^{2}$}

\section{L'équité et l'égalité des chances}

8 Pour que l'école soit une école de l'égalité des chances et non pas une institution productrice des inégalités, la Vision érige l'équité comme un fondement pour la réforme à l'horizon 2030. Instaurer le principe d'équité à l'école, c'est œuvrer en profondeur contre les disparités sociales et territoriales à travers la généralisation de l'éducation obligatoire, l'adoption de la discrimination positive envers le rural et l'obligation de la préscolarisation des enfants entre 4-5 ans. L'enseignement préscolaire, qui répond aux normes de l'éducation de la petite enfance, est limité aux grandes villes et généralement offert par le secteur privé. Il est devenu une source d'inégalité entre les enfants, entre ceux dont les familles ont les moyens de financer cet enseignement et ceux qui ne peuvent voir leurs enfants y accéder. Pour instaurer une égalité des chances, la Vision préconise son obligation pour la petite enfance.

On ne peut mettre en œuvre le principe d'équité et d'égalité des chances sans envisager de mettre les enfants à un même niveau de capital culturel souvent acquis grâce à l'environnement social. La réforme recommande un soutien scolaire pour les élèves qui se trouvent fragilisés dans le milieu scolaire, une éducation inclusive des enfants handicapés, et un développement de l'attractivité de l'école par les activités culturelles, artistiques, numériques et sportives pour créer un environnement susceptible de réduire les disparités. Ceci contribuera à la démocratisation de l'éducation, favorisera l'égalité des chances et permettra d'atténuer de manière substantielle le fossé qui se creuse entre une élite sociale qui protège ses enfants, en les plaçant dans des écoles privées pour en faire des privilégiés, et le reste des couches sociales, généralement défavorisées, qui optent par défaut pour l'école publique.

10 Les principes d'équité et de l'égalité des chances, placés comme fondement de la nouvelle réforme dans une société avec des disparités sociales, vise à faire de l'école un vecteur producteur du lien social.

\section{Euvrer au profit d'une école de la qualité}

11 Soumettre l'éducation à l'exigence de la qualité constitue un des fondements de la Vision stratégique. La mesure et l'évaluation de la qualité, à travers les enquêtes internationales, les audits, et les accréditations des programmes et des établissements, mettent à l'épreuve l'école et l'université. S'agissant des acquis des élèves marocains, leur niveau en $4^{\mathrm{e}}$ année du primaire, entre 2003 et 2011, a reculé en mathématiques et en sciences et n'atteint pas la moyenne en langues ${ }^{3}$. Plusieurs facteurs contribuent à la 
dégradation du niveau des acquis: la non généralisation du préscolaire, le retard scolaire, l'environnement pédagogique, le taux d'encadrement, la charge horaire des enseignants, l'état des établissements, le non encadrement de l'accès aux technologies de l'information et de la communication (TIC) et l'environnement socioculturel de la famille.

La Vision appelle implicitement à changer de paradigme éducatif. Cela implique un travail de redéfinition du socle des connaissances à faire acquérir aux élèves pour chaque cycle, et perceptible de manière positive dans l'évaluation de leurs acquis; l'instauration de l'innovation pédagogique dans les classes en personnalisant les apprentissages par l'adoption de méthodes différenciées pour chaque élève; l'adaptation du rythme scolaire à l'environnement de l'école, notamment en milieu rural. Dans ce cadre, la Vision recommande une réforme centrée autour de l'apprenant avec une rénovation des méthodes pédagogiques au niveau de la classe.

Par ailleurs, l'accès à la formation professionnelle doit être lié à l'orientation avec l'adoption de mesures de flexibilité en mettant en place des passerelles. Au niveau pédagogique, il faudrait articuler les enseignements théoriques aux pratiques et favoriser l'enseignement par alternance avec l'entreprise.

La Vision définit les choix linguistiques en accordant à chaque langue (l'arabe et l'amazigh, ainsi que les langues étrangères) un positionnement dans le système d'éducation et de formation. Elle instaure une cohérence linguistique dans une perspective d'équilibre durable entre le principe de justice linguistique et l'impératif de la maîtrise des langues nationales et étrangères. Elle recommande une nouvelle architecture pour l'enseignement des langues et des langues d'enseignement. Tout en consolidant le rôle primordial des langues arabe et amazigh, langues nationales constituant les fondements de l'identité marocaine comme langues d'enseignement et langues enseignés, la réforme positionne dans l'enseignement deux autres langues : le français, à partir de la première année du primaire, et l'anglais, à partir de la quatrième année, comme langues d'ouverture sur l'international. La Vision invite également à renforcer et à moderniser les langues nationales en lien avec la rénovation des méthodes pédagogiques, dans le cadre de l'élaboration d'un plan national pour le développement de ces langues.

15 C'est ainsi que la réforme consolide le choix d'un pluralisme linguistique assumé et affirmé, tout en proposant une architecture du positionnement des langues tout au long du cursus, du préscolaire à l'enseignement supérieur. Ce pluralisme linguistique correspond à la pluralité inhérente à la culture marocaine, une pluralité sanctuarisée par la Constitution de 2011, qui fait de l'identité marocaine une identité plurielle.

Par ailleurs, le métier d'enseignant connaît des évolutions importantes partout dans le monde. Ces dernières sont liées aux changements survenus au niveau de la génération actuelle des apprenants ${ }^{4}$ : mutations dans la culture des jeunes, attentes de la société, évolution des méthodes d'apprentissage et développement des TIC, apparition de contenus numériques éducatifs, etc., sont autant de facteurs qui doivent déterminer le nouveau profil du métier. Ainsi, l'enseignant passe d'un rôle traditionnel de pourvoyeur de connaissances ou d'instructeur à celui d'animateur et de facilitateur, qui doit susciter la curiosité des apprenants et leur communiquer l'art de chercher des solutions aux problèmes posés. L'enseignant d'aujourd'hui doit avoir des compétences qui lui permettent de rénover les méthodes d'apprentissage, d'animer la classe, de tuteurer les élèves dans l'usage des TIC. 
17 En raison du rôle que joue la recherche scientifique dans le développement du pays et dans son positionnement par rapport à la société de la connaissance, la Vision recommande d'instaurer une politique proactive dont les orientations visent à enraciner la culture scientifique dès le niveau primaire, de valoriser la recherche et ses résultats, de renforcer la pluridisciplinarité et la complémentarité entre sciences fondamentales et sciences appliqués, ainsi que de créer des pôles économiques et technologiques qui impliquent un partenariat entre universités, centres de recherche et entreprises.

18 La gouvernance a été identifiée par l'évaluation comme un nœud du dysfonctionnement du système d'éducation et de formation. Pour y remédier, il faudrait instaurer la cohérence d'un système d'éducation et de formation qui fonctionne en sous-systèmes, tout en érigeant le rôle régulateur de l'État à travers ses ministères.

La réforme doit renforcer l'autonomie des académies et des universités. La centralisation n'a pas favorisé la dynamisation des liens entre services centraux et entités décentralisées. Ce qui maintient la verticalité des décisions, du haut vers le bas, et laisse peu de marge pour l'autonomie des académies et des universités et pour la responsabilisation des acteurs régionaux et locaux. La Vision recommande donc un renouvellement des modes de gouvernance et de conduite du changement, avec un leadership efficace capable de mettre en œuvre les réformes et de les guider grâce à de nouvelles méthodes, tout en décentralisant et en responsabilisant les leaders régionaux. Il faudrait par ailleurs instaurer des mécanismes de veille, de suivi et d'évaluation tout en mettant en place des modalités de pérennisation et de diversification des ressources financières et en encourageant la mobilisation de tous, à travers l'élaboration d'un pacte national au profit de l'école.

20 En visant la qualité des apprentissages et des méthodes pédagogiques pour améliorer les acquis des élèves, la formation des lauréats, la formation initiale et continue des étudiants, la Vision préconise l'amélioration de la performance du système d'éducation et de formation ainsi que le rendement interne et externe des écoles et des universités, en œuvrant pour une gouvernance de qualité, véritable courroie de transmission du fonctionnement du système éducatif.

\section{Une école de l'émancipation de l'individu et du développement de la société}

21 La Vision prône une école émancipatrice de l'individu. Il est évident que la valorisation des citoyens passe par la qualité de l'éducation et de la formation et par leur capacité à s'adapter à une société en plein changement et aux métiers nouveaux d'avenir, avec notamment le développement accéléré des technologies de l'information. L'intégration professionnelle et l'accès à l'emploi sont tributaires des formations combinant les versants académique et pratique, et de formations en alternance avec les milieux professionnels, tout en dotant les diplômés d'ouverture d'esprit et de capacités d'initiative.

22 L'école et l'université sont également des vecteurs du développement de la société dans le cadre d'une société égalitaire et démocratique. Elles consolident cette fonction lorsqu'elles véhiculent et transmettent à toute une génération les valeurs des droits 
humains, le sens civique, les valeurs démocratiques, l'égalité entre les hommes et les femmes, le respect de l'environnement et assurent la formation tout le long de la vie. La Vision appelle ainsi à «nettoyer » les manuels scolaires des stéréotypes sur les femmes et invite à une réforme des contenus d'enseignement.

L'école doit œuvrer pour que les élèves s'approprient les valeurs du vivre ensemble, quelles que soient leur appartenance sociale, ethnique et religieuse et leur affiliation politique ; elle doit leur transmettre des valeurs créatrices du lien social et les doter de capacités à intégrer l'évolution de la société et du monde.

La Vision recommande le développement de l'université pour qu'elle soit un lieu de formation du savoir, de la recherche et de l'innovation capable d'intégrer les étudiants dans la société du savoir. L'université marocaine a une double responsabilité envers la société : la première est sociale et développementale, la seconde est universelle. Cette dernière fait de l'université un espace de jonction avec l'international, pour l'actualisation des connaissances, le transfert de la technologie et des savoir-faire pédagogiques.

La Vision invite à une école à l'ambition rehaussée, capable d'assumer sa fonction libératrice et émancipatrice de l'individu afin de le préparer à son avenir et à celui du pays.

\section{Conduire le changement avec un leadership efficace}

Les réformes éducatives sont portées par des hommes et des femmes, véritables acteurs et agents du changement; d'où le rôle actif que pourraient jouer ces acteurs dans les processus d'amélioration de la qualité de l'éducation. Ainsi, la Vision recommande de proclamer la période 2015-2030 comme une période de la rénovation de l'école et de la positionner comme une priorité nationale, afin de mobiliser la Nation autour de son école. Par ailleurs, pour lui assurer une continuité au-delà des vicissitudes de conjoncture, il est recommandé de la consigner dans une loi-cadre ${ }^{5}$.

La mise en place des réformes et leur réussite sont tributaires de la conduite du changement et d'un leadership capable de mobiliser, de piloter et de guider tout en faisant participer et en impliquant les acteurs pédagogiques et toutes les parties prenantes : parents d'élèves, collectivités locales, ONG, entreprises... Il faut s'appuyer sur des compétences en capacité de créer une dynamique mobilisatrice autour de l'éducation.

28 L'école marocaine se trouve aujourd'hui face à des défis dus aux impératifs socioéconomiques d'une société en développement et aux exigences d'une globalisation qui impacte l'école et tout le système d'éducation et de production du savoir. Ceci interpelle l'État pour qu'il renouvelle l'école et ses missions, en conformité avec le projet de société porté par la nouvelle Constitution.

La Vision stratégique 2015-2030 annonce une école renouvelée. Certes les changements ont toujours été liés à l'évolution des savoirs et des connaissances, aux progrès de la science et des savoir-faire, et la nécessité d'introduire des réformes a toujours fait partie de l'évolution de l'éducation et de l'école. Néanmoins, la situation de l'éducation dans la société marocaine impose un sursaut collectif et une mobilisation de la Nation.

Le caractère affirmé de ces réformes n'en fait pas de simples mesures de conjoncture. Il s'agit d'un changement de paradigme qui inscrit le développement de l'école et de 
l'université marocaines dans une temporalité longue et pérenne, afin de garantir une performance et des effets positifs, progressivement tangibles, en ce qui concerne les acquis des élèves et les qualifications des diplômés. Mais il faut aussi envisager les obstacles que peut rencontrer une réforme qui se voudrait globale. L'histoire des tentatives de réforme successives et inachevées qu'a connues l'éducation au Maroc plane sur toute nouvelle réforme. Le défi est d'arrimer une Vision stratégique ambitieuse, capable d'intégrer les réponses aux attentes exprimées par les diverses parties prenantes - familles, acteurs pédagogiques, secteur économique, médias -, une prise de conscience collective des déficits de l'école, à la capacité de la gouvernance à traduire cette Vision en mesures et actions opérationnelles qui pourront générer de la performance et conduire les élèves vers la réussite.

\section{NOTES}

1. Instance nationale d'évaluation auprès du Conseil supérieur de l'éducation, la formation et la recherche scientifique (2014): Rapport analytique. Mise en œuvre de la Charte nationale d'éducation et de formation 2000-2013. Acquis, enjeux et défis. Rabat Voir également: Atlas graphique et cartographique de la décennie de l'éducation et de la formation 2000-2013 [http://www.csefrs.ma/pdf/ ATLAS_Graphique.pdf]. Voir site du Conseil (en arabe et en français) : www.csefrs.ma.

2. CSEFRS : Pour une école de l'équité, de la qualité et de la promotion. Vision stratégique de la réforme 2015-2030, Rabat, 2015. Voir site du Conseil (en arabe et en français) : www.csefrs.ma.

3. Comme le montrent les enquêtes internationales TIMMS et PIRLS conduites en 2011 par l'International Association for the Evaluation of Educational Achievement (IEA).

4. Voir S. Clerc et Y. Michaud (2010), Face à la classe, Gallimard.

5. Au moment de la rédaction de cet article, une commission interministérielle travaille sur l'élaboration de cette loi-cadre.

\section{INDEX}

Mots-clés : égalité des chances, qualité de l'éducation, réforme de l'enseignement, équité, leadership

Palabras claves : igualdad de oportunidades, calidad de la educación, reforma de la educación, equidad, liderazgo

Index géographique : Maroc

Keywords : equal opportunity, educational quality, educational reform, equity, leadership 


\section{AUTEUR}

\section{RAHMA BOURQIA}

Rahma Bourqia est sociologue et professeur des universités. Elle a, entre autres, été présidente de l'Université Hassan II de Mohammédia 2002-2010. Elle a publié sur les thématiques suivantes : l'État, les femmes, les jeunes et la culture politique. Elle était membre du conseil d'administration de l'Université des Nations unies Tokyo (2010-2016), membre fondateur et présidente du Conseil arabe des sciences sociales Liban (2005-2014), membre du bureau de l'Association internationale des sociologues de langue française (AISLF). Elle est aujourd'hui directrice de l'instance d'évaluation du Conseil supérieur de l'éducation, de la formation et de la recherche scientifique (CSEFRS) du Maroc. Courriel : bourqiarahma@gmail.com 